\title{
El Aprendizaje Inicial de la Lectura y la Escritura de Palabras en Español: Un Estudio de Caso
}

\section{Beginning Reading and Spelling Acquisition in Spanish: A Case Study}

\author{
Beatriz Diuk \\ Consejo Nacional de Investigaciones Científicas y Técnicas \\ Universidad Nacional de San Martín
}

\begin{abstract}
El presente es un estudio de caso en el que se analizó el proceso de aprendizaje de la lectura y la escritura de palabras en dos niñas de 1er año de Educación General Básica. Las niñas provenían de familias de sectores marginados de Buenos Aires, Argentina y participaron, a contraturno de la escuela, de un programa de alfabetización intercultural. El estudio en detalle a lo largo de un año del proceso de adquisición de lectura y escritura permitió identificar las estrategias utilizadas por las niñas en distintos momentos del aprendizaje. Asimismo se obtuvo evidencia sobre el impacto que las propuestas de enseñanza de las maestras tenían sobre la adopción de estas estrategias, en particular sobre el uso de la recodificación fonológica.
\end{abstract}

Palabras Clave: aprendizaje, estrategias de lectura, estrategias de escritura, nivel socioeconómico bajo, intervención pedagógica.

\begin{abstract}
The aim of this study was to analyze the reading and spelling acquisition process of two girls attending the 1st year of General Basic Education. The girls came from low-income families of Buenos Aires, Argentina and they attended an after-school intercultural literacy program. The longitudinal study made it possible to identify the reading and spelling strategies used by the girls and the difficulties they found in the process of literacy acquisition. Additionally, evidence was obtained concerning the impact of the teaching strategies in the acquisition process. The effect of systematic instruction in phonological recoding was particularly evident.
\end{abstract}

Keywords: reading strategies, spelling strategies, low-income children, instruction programs, learning.

El presente trabajo es parte de un proyecto más amplio que tiene como objetivo analizar las dificultades que enfrentan los niños de sectores marginados en el aprendizaje de la lectura y la escritura de palabras. Es un hecho reconocido que entre estos niños se producen los mayores índices de fracaso escolar, fracaso que está asociado a la falta de oportunidades educativas y que se manifiesta en las dificultades que los niños encuentran en su proceso de alfabetización.

La magnitud de la problemática del fracaso escolar y su estrecha asociación con la procedencia socioeconómica de los niños indican que los bajos niveles de aprendizaje no pueden ser atribuidos a problemas específicos de los propios niños. En efecto, se ha estimado que la prevalencia de las dificulta-

Beatriz Diuk, Consejo Nacional de Investigaciones Científicas y Técnicas, y Universidad Nacional de San Martín de Argentina.

La correspondencia relativa a este artículo deberá ser dirigida a la autora a Tte. Gral. Perón 2158 (C1040AAH), Buenos Aires, Argentina. E-mail: bdiuk@dklab.com des lectoras de origen constitucional no supera el $8 \%$ de la población (Stanovich, 2000), un porcentaje muy inferior al de los niños marginados que enfrentan problemas en la adquisición de la lectura. En consecuencia, las dificultades de estos niños sólo pueden ser atribuidas a factores contextuales, en particular, a la falta de oportunidades para el aprendizaje (Capovilla \& Capovilla, 2004).

Ahora bien, para poder dar una respuesta adecuada a la problemática de los sectores marginados resulta necesario conocer en profundidad el proceso de aprendizaje de la lectura y la escritura en estos niños y explorar la naturaleza de las dificultades que muchos de ellos enfrentan. Para estudiar estos procesos, se han tomado como referencia los modelos de adquisición que han sido desarrollados en el marco de la psicología cognitiva (Ehri, 1991, 1999; Frith, 1985). Los modelos más recientes reconocen el recurso a una serie de estrategias progresivamente más avanzadas para leer palabras: una estrategia no analítica o logográfica, una estrategia analítica parcial, una estrategia analítica completa o avanza- 
da y una estrategia analítica consolidada. El progreso en el aprendizaje está relacionado con el desarrollo de la conciencia fonológica - esto es, de la capacidad para analizar explícitamente la estructura fonológica de las emisiones orales-y con el conocimiento de las correspondencias grafema-fonema.

La estrategia logográfica es utilizada en los primeros momentos del aprendizaje de la lectura, cuando los niños identifican palabras conocidas a partir de pistas visuales salientes que han aprendido a asociar con el significado de la palabra, sin relación con la forma fonológica. El recurso a una estrategia analítica parcial se produce cuando los niños aprenden algunas correspondencias grafema-fonema y adquieren habilidades incipientes de conciencia fonémica que les permiten atender a algunas letras de las palabras. Los niños utilizan estas letras como pistas para establecer conexiones sistemáticas, aunque parciales, entre la palabra escrita y su pronunciación, dando lugar a frecuentes lecturas erróneas de palabras desconocidas que son confundidas con palabras conocidas con las que comparten letras. Los niños que utilizan una estrategia analítica completa poseen habilidades de síntesis fonológica y un conocimiento operativo del principio alfabético que les permiten reconocer palabras escritas y acceder a su significado. Este reconocimiento se produce a través de la recodificación fonológica de las palabras, mecanismo que consiste en obtener el significado de una palabra escrita mediante la recodificación de los signos gráficos en una representación sonora. Los niños comienzan a utilizar una estrategia analítica consolidada cuando desarrollan la habilidad de procesar patrones ortográficos mayores que el grafema. Asimismo, se establecen conexiones específicas por palabra. Como consecuencia, las palabras son leídas en forma más veloz y fluida.

Con respecto al aprendizaje de la escritura, Ellis (1994) sintetiza los resultados de distintos trabajos en un modelo que incorpora una serie de etapas: (a) etapa precomunicativa, en la que los niños producen secuencias de letras elegidas al azar; (b) etapa semifonética, en la que las escrituras representan parte de los sonidos de las palabras; (c) etapa fonética, en la que todos los sonidos de las palabras están representados; y (d) las etapas transicional y correcta, en las que los niños avanzan en el dominio de la ortografía. Ellis (1994) considera que este modelo detallado del progreso en la adquisición de la escritura se corresponde globalmente con modelos como el de Frith (1985), que incluyó tres etapas: logográfica, alfabética y ortográfica.
Una característica particularmente relevante del modelo de Frith reside en el hecho de que incorpora la relación entre el aprendizaje de la lectura y el de la escritura. Frith (1985) postula que la adquisición de la escritura atraviesa la misma serie de etapas que la de la lectura pero que el desarrollo de estas etapas se produce de forma asincrónica: el uso de una estrategia logográfica se adopta antes para la lectura que para la escritura en tanto la estrategia analítica comienza a desarrollarse en la escritura y luego se transfiere a la lectura. La adquisición de una estrategia ortográfica, esto es, una estrategia que implica el procesamiento de patrones ortográficos mayores que el grafema, es anterior en la lectura que en la escritura.

Ahora bien, estos modelos han sido desarrollados para explicar la adquisición de la lectura y la escritura en inglés. El inglés, a diferencia del español, es una lengua de ortografía opaca. La transparencia u opacidad de un sistema ortográfico hace referencia al mayor o menor grado de consistencia entre la representación fonológica y la representación ortográfica. En inglés, las relaciones G-F y F-G son sumamente complejas por lo que los grafemas tomados individualmente no tienen representaciones fonológicas invariantes (Stanovich, 2000). El español, en cambio, es una de las lenguas de ortografía más transparente. Si bien las correspondencias son más consistentes en la dirección grafemafonema, proporcionando en este sentido cierta ventaja para la lectura, también las correspondencias fonema-grafema presentan pocas irregularidades.

Distintos investigadores han sostenido que las características de la lengua inciden en los mecanismos iniciales de procesamiento de palabras dado que las diferentes ortografías imponen distintas demandas al lector principiante (Wimmer \& Goswami, 1994). En una ortografía como la del inglés, al inicio del aprendizaje el niño recurrirá a mecanismos de asociación entre patrones visuales no analíticos y sus pronunciaciones. En cambio, si una ortografía es consistente en cuanto a las relaciones entre escritura y sonido, las correspondencias G-F serán más fáciles de descubrir y usar. Investigaciones realizadas en español han mostrado que los niños manifiestan tempranamente una tendencia mayor a apoyarse en estrategias de procesamiento fonológico (Signorini, 1997, 1999; Signorini \& Piacente, 2001). La comparación entre niños que aprenden a leer lenguas de distinta profundidad ortográfica ha mostrado una ventaja en términos de velocidad de adquisición para las ortografías transparentes, asociada a 
una ausencia comparativa del recurso al procesamiento léxico (Goswami, Gombert \& Fraca da Barrera, 1998). El sesgo hacia el procesamiento fonológico se verifica incluso en los niños con dificultades específicas para la lectura quienes logran, en pocos años, leer palabras en forma precisa (Wimmer, 1993).

Ahora bien, en un estudio previo realizado con niños de nivel socioeconómico (NSE) bajo, se encontró evidencia del recurso persistente a mecanismos no fonológicos (Diuk, Signorini \& Borzone, 2003). Cuando se comparó el desempeño lector de un grupo de niños de NSE bajo de 3er año de Educación General Básica con el de niños de NSE medio de menor edad equiparados por nivel de lectura, los niños de NSE bajo pusieron de manifiesto una mayor tendencia a producir errores de lectura consistentes en responder con palabras diferentes de aquellas que se les habían presentado. Diversas investigaciones han mostrado que, cuando los niños comienzan a recodificar fonológicamente las palabras, los errores que producen dan lugar a pseudopalabras, no a palabras distintas de las presentadas (Signorini, 1997). El alto porcentaje de respuestas palabra en los niños estudiados llevó a considerar que estaban utilizando una estrategia de recodificación lexicalizada (FletcherFlinn \& Thompson, 2000). El análisis de los errores mostró que, al menos en algunos casos, los niños no lograban procesar todos los grafemas de la palabra por lo que la recodificaban parcialmente y luego recurrirían a información léxica para completar la pronunciación del estímulo, compensando con mecanismos léxicos la relativa ineficacia de la estrategia analítica. Los niños parecían no poder aprovechar las ventajas que ofrece la ortografía transparente del español y recurrían a mecanismos léxicos con una frecuencia similar a la que se encontró en estudios realizados con niños que aprenden a leer en inglés (Frith, Wimmer \& Landerl, 1998).

En el presente trabajo nos propusimos realizar un estudio de caso en el que se analizara en detalle, a lo largo del primer año de escolarización, el proceso de aprendizaje de la lectura y la escritura en dos niñas que vivían en contextos de pobreza. Se esperaba que este análisis contribuyera a especificar los mecanismos que determinaban las características del proceso lector identificado en estudios previos. Asimismo, dada la naturaleza experiencial de las dificultades de los niños marginados, nos interesaba analizar el impacto del proceso de enseñanza sobre la conformación del patrón de aprendizaje.
En consecuencia, se estudió el proceso de aprendizaje de niños que participaban de la implementación de una propuesta de alfabetización que incorpora sistemáticamente situaciones para promover el desarrollo de la conciencia fonológica y el aprendizaje de las correspondencias fonema-grafema. El énfasis en el desarrollo de la conciencia fonológica se relaciona con la evidencia acerca de una relación causal entre este desarrollo y el aprendizaje de la lectura y la escritura en un gran número de lenguas de ortografía alfabética (Borzone \& Signorini, 1994; Bravo, Villalón \& Orellana, 2002; Cardoso-Martins, 1995). Asimismo, los resultados de diversas investigaciones han mostrado que los niños que crecen en contextos de pobreza ingresan a la escuela con un menor desarrollo de las habilidades de conciencia fonológica si se los compara con niños provenientes de sectores más aventajados (Adams, 1990; Borzone, 1997).

En consecuencia, la intervención pedagógica proporcionaba a los niños un contexto más favorable para el aprendizaje y en ese marco se estudió, a lo largo de un año, el proceso de adquisición de la lectura y la escritura.

\section{Método}

\section{Participantes}

Participaron del estudio dos niñas cuyas familias vivían en un barrio marginado del conurbano de Buenos Aires. Ambas niñas asistían, a contraturno de la escuela, a un centro comunitario de educación complementaria donde participaron de una propuesta de intervención pedagógica junto con otros 9 niños de 1 er año, 7 de 2 do y 8 de 3ero. Se realizó un seguimiento anual del proceso de aprendizaje de todos los niños de 1er año. Las niñas presentadas aquí fueron seleccionadas por presentar, al finalizar el año, un nivel de desempeño correspondiente a la media de su grupo.

Una de las niñas, Natalia, contaba con 5 años y 9 meses al incorporarse al estudio. Cursaba 1er año de EGB en una escuela pública cercana al barrio. El año anterior Natalia había concurrido al preescolar en un jardín de infantes oficial y había participado de un grupo de educación preescolar que funcionaba 3 veces por semana en el centro comunitario.

La otra niña, Inés, tenía 6 años y 8 meses. Inés había vivido los primeros años de su vida junto con su abuela en el interior del país. A mediados del año anterior la madre la había traído a Buenos Aires para que viviera con ella. En este proceso su primer año de escolarización había quedado inconcluso y estaba cursando nuevamente el 1er año de EGB.

La propuesta de intervención pedagógica. Ambas niñas participaban, en el centro comunitario al que asistían, de una experiencia de alfabetización intercultural (Diuk, Borzone \& Rosemberg, 2000).

La propuesta se basa en una concepción de los procesos de enseñanza y aprendizaje en la interacción social y articula distintas dimensiones de conocimiento: conocimiento sobre el proceso de alfabetización, proporcionado por los estudios 
realizados en el marco de la psicología cognitiva; conocimiento sobre los procesos de enseñanza y aprendizaje encuadrado en la teoría socio-histórica y cultural, y conocimiento sobre el mundo social y cultural de los niños.

En base a estas dimensiones de conocimiento se estructuran las situaciones de enseñanza y aprendizaje en el aula, en las que se integra el trabajo con el lenguaje oral y escrito y se articulan la producción y lectura de textos en el marco de experiencias comunicativas variadas con la enseñanza explícita y sistemática de la lectura y la escritura de palabras. En particular, la propuesta enfatizaba la implementación de situaciones para promover el desarrollo de la conciencia fonológica.

\section{Materiales y Procedimiento}

Al comenzar el año todos los niños de 1er año del centro comunitario fueron evaluados mediante pruebas que medían sus habilidades de conciencia fonológica -reconocimiento de rimas y de sonido inicial, síntesis de sonidos-, el conocimiento de las letras y las habilidades de lectura y escritura de palabras. Para el estudio en detalle de las estrategias de lectura y escritura a lo largo del año se elaboraron pruebas adicionales de lectura y escritura de palabras. Al finalizar el año se administró una prueba de lectura y de escritura de palabras.

En todos los casos las pruebas fueron administradas en un espacio tranquilo en el centro comunitario al que concurrían los niños. Las pruebas administradas al comenzar y al finalizar el año fueron grabadas en audio. Las pruebas utilizadas durante el año para evaluar el progreso en el uso de las estrategias de lectura y escritura fueron grabadas en video.

El estudio longitudinal de las estrategias comenzó a mediados del mes de marzo y las niñas fueron evaluadas en forma quincenal a lo largo de las 35 semanas de clases. Se realizaron 14 sesiones con Natalia y 17 sesiones con Inés. En cada sesión se les solicitaba la lectura y la escritura de una serie de palabras. Asimismo, las niñas fueron estimuladas a verbalizar las estrategias que empleaban al leer o al escribir. Diversas investigaciones han mostrado la validez de este procedimiento para conocer las estrategias utilizadas por los niños (Siegler, 1991; Steffler, Varnhagen, Friesen \& Treiman, 1998).

\section{Pruebas administradas al comenzar el año.}

- Reconocimiento de rima: se trata de una prueba de opción múltiple elaborada por Signorini y Borzone de Manrique (1996). La prueba está formada por 10 ítemes. El puntaje se determina por el número de aciertos. El coeficiente de confiabilidad (Spearman-Brown) fue de .80 .

- Identificación de sonido inicial: la prueba presenta la misma estructura que la prueba de reconocimiento de rima. El coeficiente de confiabilidad fue de. 75 .

- Síntesis de sonidos: en esta prueba, la entrevistadora articula en forma separada los sonidos de una palabra y se pide al niño que identifique de qué palabra se trata y la pronuncie. La prueba está formada por 20 ítemes: seis de dos segmentos, siete de tres segmentos y siete de cuatro segmentos. El puntaje es el número de respuestas correctas. El coeficiente de confiabilidad fue de .77.

- Reconocimiento de letras: se pidió a los niños que nombraran 22 letras del abecedario presentadas individualmente en cartones en imprenta mayúscula. Se consideró que el niño reconocía la letra si proporcionaba el nombre, el sonido o una palabra que comenzara con dicha letra. El coeficiente de confiabilidad fue de .90 .

\section{Pruebas administradas al comenzar $y$ al finalizar el año.}

- Escritura y lectura de palabras: se trata de una prueba formada por 32 palabras distribuidas en tres series. Las 10 palabras de la primera serie son bisílabas, con estructura silábica CV. La segunda serie, de 10 palabras, incorpora palabras bisílabas con estructuras silábicas más complejas: diptongos, grupos consonánticos y palabras con sílabas CVC. En la siguiente serie las 12 palabras cuentan con tres y cuatro sílabas: cuatro palabras tienen sílabas con estructura $\mathrm{CV}$ en tanto las restantes incorporan diptongos, grupos consonánticos y consonantes en posición implosiva. Al comenzar el año se administró la primera serie de la prueba tanto para la lectura como para la escritura. Al finalizar el año se aplicó la prueba completa. Para la asignación de puntaje en la escritura se utilizó una escala entre 0 y 5 que evaluaba el grado de completamiento de las palabras (Borzone \& Diuk, 2001). En la lectura se asignó un punto por cada palabra leída sin errores de pronunciación. El coeficiente de confiabilidad fue de .96 para la lectura y de .99 para la escritura.

- Lectura de pseudopalabras: al finalizar el año se administró asimismo una prueba de lectura de pseudopalabras. La prueba está formada por 20 pseudopalabras de dos y tres sílabas. El coeficiente de confiabilidad fue de .92 . 
Pruebas administradas durante el año para el estudio en detalle de las estrategias de lectura y escritura de palabras. Se elaboraron dos pruebas que fueron utilizadas para la evaluación tanto de la lectura como de la escritura de palabras aisladas.

La primera prueba fue elaborada con el objeto de identificar el uso de una estrategia logográfica y de lectura por pista fonética. La prueba constaba de tres series de palabras. La primera serie incluía palabras seleccionadas del abecedario mural del aula del centro comunitario, palabras que, por ello mismo resultaban muy familiares para los niños. La segunda serie estaba conformada por palabras menos familiares que comenzaban con la misma letra y tenían la misma longitud que las palabras familiares de la serie 1 . Así por ejemplo, la serie 1 incluía zapato y la serie 2, zapallo. Las palabras de la tercera serie también comenzaban con la misma letra pero presentaban mayores diferencias con las palabras de la serie 1. Para la escritura se utilizó parte de las palabras de las series de lectura, presentadas en un orden diferente.

Cuando las niñas avanzaron en su desempeño hacia una estrategia analítica, se elaboró una nueva prueba de lectura de palabras conformada por dos series de palabras. En la primera serie todas las palabras, de longitud creciente, estaban formadas por sílabas con estructura CV. Las palabras de la segunda serie presentaban estructuras silábicas más complejas.

\section{Resultados y Discusión}

\section{Natalia $^{1}$}

En la Tabla 1 se presenta el desempeño del grupo al que pertenecían las niñas estudiadas así como los puntajes obtenidos por cada una de ellas. Tal como se observa, en las evaluaciones iniciales Natalia obtuvo puntajes superiores a la media de su grupo en las tareas de reconocimiento de rima, de identificación de sonido inicial y en las de lectura y escritura de palabras. El desempeño en la tarea de síntesis de sonidos coincidió con el puntaje promedio. Reconoció todas las letras que se le presentaron (ver Tabla 1).

Al comenzar el estudio de las estrategias de lectura y escritura, Natalia recurría a una estrategia analítica parcial para reconocer las palabras escritas. El uso de esta estrategia pudo inferirse del hecho de que los errores de la niña consistían en palabras que compartían algún grafema con el estímulo presentado pero diferían en los grafemas restantes.

Este desempeño era esperable en función de los resultados de las evaluaciones iniciales. Natalia pudo identificar el sonido inicial de la mitad de los estímulos presentados y nombró todas las letras, conocimientos necesarios para reconocer palabras utilizando pistas fonéticas (Ehri, 1991). Natalia carecía, en cambio, de habilidades de síntesis fonológica, habilidades requeridas para el desarrollo de una estrategia analítica completa (Perfetti, 1992).

Tabla 1

Puntajes obtenidos por el grupo de ler año y por las niñas participantes en el presente estudio en las pruebas administradas en el mes de marzo (expresados en porcentajes)

\begin{tabular}{|c|c|c|c|c|}
\hline Variable & Media grupal & Mediana & Natalia & Inés \\
\hline Rima & $63.33(30.82)$ & 80 & 90 & 80 \\
\hline Sonido inicial & $26.67(13.26)$ & 20 & 50 & 20 \\
\hline Síntesis de sonidos & $15.88 \quad(5.38)$ & 15 & 15 & 25 \\
\hline Reconocimiento de letras & $44.00(32.50)$ & 32 & 100 & 32 \\
\hline Lectura de palabras & $16.60(12.16)$ & 12 & 17 & 14 \\
\hline Escritura de palabras & $35.29(13.88)$ & 31 & 53 & 53 \\
\hline
\end{tabular}

1 Los nombres de las niñas son seudónimos similares a los nombres reales. 
Con respecto a la escritura, Natalia podía escribir en forma completa algunas palabras muy familiares. En palabras menos frecuentes su desempeño era inferior (ver Tabla 2).

Tabla 2

Escrituras producidas por Natalia en el mes de abril

\begin{tabular}{ll}
\hline Palabra dictada & Escritura \\
\hline mamá & MAMA \\
sol & SOL \\
oso & OSO \\
gato & GAO \\
uva & UA \\
estrella & ESA \\
\hline
\end{tabular}

La comparación entre las distintas formas de escritura sugiere que Natalia utilizaba una estrategia logográfica para las palabras más familiares en tanto adoptaba una estrategia semifonética en aquellas palabras cuya forma gráfica desconocía. En estas últimas, el grado de completamiento de las escrituras estuvo asociado a la longitud de los estímulos.

El desempeño de Natalia al escribir mejoró sostenidamente a lo largo del año, al consolidar el dominio de una estrategia fonética. En el mes de agosto, tal como se observa en la Tabla 3, Natalia escribía sin omisiones las palabras de dos sílabas con estructura silábica simple (consonante-vocal). Se había reducido el efecto del largo en su desempeño, pero continuaba presentando dificultades en sílabas de estructura compleja (CVC o CCV).

El uso de una estrategia fonética se manifestaba también en el autodictado que Natalia realizaba para apoyar su proceso de escritura, particularmente en el caso de las palabras poco frecuentes o en las palabras con estructura silábica compleja. Es posible observar que los fonemas efectivamente represen- tados son aquellos que fueron prolongados oralmente.

La utilización de una estrategia analítica en la escritura contrastaba con el desempeño de la niña al leer. En la misma fecha en que produjo las escrituras anteriores, Natalia recurría a la lectura por pista fonética para intentar reconocer palabras (ver Tabla 4). Los errores de Natalia consistían en pronunciar palabras que compartían el grafema inicial con el estímulo presentado pero diferían en los grafemas restantes. Ante algunas palabras se negaba a leer, comportamiento frecuente entre los niños que carecen de habilidades de recodificación fonológica (Ehri, 1991).

Tabla 4

Lectura de palabras por parte de Natalia en el mes de agosto

\begin{tabular}{ll}
\hline Palabra presentada & Lectura \\
\hline llanto & lluvia \\
guante & no sé, paso \\
nuez & nido \\
escalera & paso \\
boleto & banana \\
quiniela & queso \\
ola & paso \\
\hline
\end{tabular}

La asincronía en el desarrollo de las habilidades de escritura y de lectura está bien documentada en la literatura sobre adquisición lectora (Borzone \& Signorini, 1994; Frith, 1985). Sin embargo, en el caso de Natalia esta asincronía se prolongó varios meses, durante los cuales el desarrollo de una estrategia analítica en la escritura no contribuyó a dar lugar a una estrategia analítica en la lectura.

Tabla 3

Escritura de palabras producida por Natalia en el mes de agosto

\begin{tabular}{lll}
\hline Palabra dictada & Escritura & Pronunciación \\
\hline mesa & MESA & sin autodictado \\
loro & LORO & looooo rrrr rrrroooo \\
elefante & ELEFATE & e leeee fa ttteeee \\
estante & ETATE & eeeeees taaaaan tteeee \\
\hline
\end{tabular}


Esta asincronía, que también se presentaba en otros niños del grupo, condujo a un replanteo de las estrategias didácticas utilizadas en la propuesta de intervención. En efecto, las situaciones implementadas hasta el momento habían enfatizado el trabajo de segmentación fonológica acompañada de la escritura de palabras. Las situaciones de lectura, en cambio, tendían a promover un reconocimiento global de las palabras dado que se realizaban fundamentalmente lecturas repetidas e identificación de palabras en textos, identificación que podía realizarse en base a pistas fonéticas. Es posible pensar que la diferencia en el abordaje didáctico de la lectura y de la escritura incidía en las estrategias cognitivas de los niños. En consecuencia, se decidió introducir el modelado explícito de la recodificación fonológica mediante un juego.

Ocho días después de la introducción de este juego Natalia produjo, por primera vez, una pseudopalabra al intentar leer, comportamiento que es indicador del uso de la recodificación fonológica. Al presentársele la palabra uno, Natalia pronunció: nua.

En la misma sesión, cuando se le pidió que leyera la palabra lona, Natalia dudó, pronunció león y miró a la entrevistadora. Se produjo el siguiente intercambio:

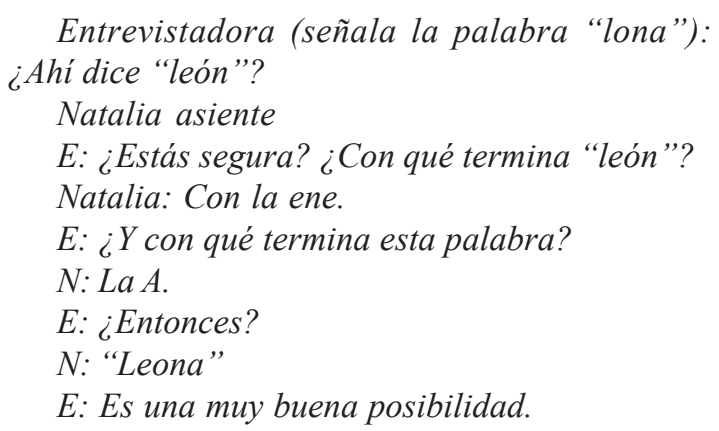

Natalia podía adicionar fonemas, pero no lograba reconocer palabras. Diversas investigaciones han mostrado que la habilidad para elidir o adicionar fonemas recién se manifiesta en los niños lectores (Cardoso-Martins, 1995). Este hecho, sumado al desempeño de Natalia al escribir, evidenciaban que había desarrollado las habilidades de procesamiento fonológico necesarias para leer. Pero no lograba recodificar las palabras.

Ahora bien, en la Tabla 5 se presentan las lecturas que Natalia realizó 15 días más tarde.
Tabla 5

Lectura de palabras por parte de Natalia a principios del mes de septiembre

\begin{tabular}{ll}
\hline Palabra presentada & Lectura \\
\hline nota & nooo ta nota \\
cola & coo la cola \\
puma & pu pu pu ppp paaa pupa. \\
& puuuu ppp ma puma \\
limón & liii mo li mo na \\
\hline
\end{tabular}

Este desempeño es propio de los niños que utilizan sistemáticamente una estrategia analítica para leer palabras. El hecho de que este progreso tuviera lugar poco tiempo después de la introducción de la enseñanza de la recodificación hace posible pensar en una relación entre ambos. Natalia presentaba pocas dificultades en las palabras con sílabas directas pero algunas dificultades persistían en las sílabas con estructura más compleja, como, por ejemplo, en la sílaba CVC en limón. La incidencia de la complejidad silábica en la lectura es considerada un indicador del uso de un mecanismo de recodificación fonológica. Este desempeño, sumado a la subvocalización que realizaba Natalia, revelan que se produjo un cambio en su estrategia de lectura de palabras.

Al finalizar el año, Natalia escribió en forma fonológicamente apropiada 26 de las 32 palabras que se le dictaron (la media grupal fue de 25) y leyó correctamente 22 de las 32 palabras que se le presentaron (la media grupal fue de 18). Natalia leyó 9 de 20 pseudopalabras, siendo la media grupal de 9.45. Estos resultados sugieren que Natalia presentaba un desempeño similar al de la media de su grupo. Cabe señalar que este grupo alcanzó niveles de lectura muy superiores a los obtenidos por niños del mismo centro comunitario evaluados en años anteriores. Así por ejemplo, en el grupo de 1er año incluido en el estudio de Diuk, Signorini y Borzone (2003) la mediana fue de 5 palabras correctamente leídas de una prueba de lectura de palabras muy similar a la utilizada en el presente estudio.

Inés

Inés ingresó al estudio con más edad y menos conocimientos que Natalia. En el mes de marzo solamente pudo reconocer 8 letras sobre las 22 que se le 
presentaron. Su desempeño en identificación de sonido inicial y en síntesis de sonidos fue cercano al piso de la prueba, aunque se desempeñó bien en el reconocimiento de rimas. El puntaje en la prueba de lectura y escritura de palabras fue cercano a la media del grupo (ver Tabla 1).

Al comenzar el estudio, Inés leía palabras mediante una estrategia analítica parcial, utilizando como pista alguna letra de la palabra presentada, tal como se ejemplifica en la Tabla 6.

Tabla 6

Lectura de palabras por parte de Inés en el mes de abril

\begin{tabular}{ll}
\hline Palabra presentada & Lectura \\
\hline uva & una \\
árbol & ala \\
sol & sapo \\
oso & oso \\
\hline
\end{tabular}

Al escribir (ver Tabla 7), Inés representaba algunos fonemas de cada palabra y completaba la escritura con letras sin relación con la palabra que intentaba producir. La producción de secuencias de letras sin relación con el estímulo ha sido identificada en español como una de las primeras formas de escritura que producen los niños pequeños (Borzone \& Signorini, 1998). Inés utilizaba, entonces, una estrategia semifonética que combinaba con una estrategia menos evolucionada. La única palabra que podía escribir en forma completa era su nombre, por lo que es posible pensar que lo hacía mediante una estrategia logográfica.

El desempeño en escritura de palabras mejoró a lo largo de los primeros meses del año, produciéndose un incremento en el número de fonemas que
Inés lograba representar en cada palabra. En el mes de junio Inés prolongaba los sonidos de las palabras al escribir, tal como se observa en la Tabla 8.

Tabla 7

Escritura de palabras producida por Inés en el mes de abril

\begin{tabular}{ll}
\hline Palabra dictada & Escritura \\
\hline gato & GDOA \\
sol & SOPSO \\
león & LETCA \\
\hline
\end{tabular}

En esa misma época, Inés continuaba utilizando una estrategia analítica parcial para leer. Pero a diferencia de Natalia, Inés reconocía que sus respuestas eran erróneas y buscaba activamente estrategias alternativas para identificar las palabras. Así por ejemplo, ante la palabra madera, Inés respondió:

I: "tomate". No. La otra. Eeeeehhh... "mamá". No, pero mamá lleva la eme, la a, otra vez la eme y otra vez la a. Lleva acento también. Por eso no sé. No lo veo por ninguna parte el acento.

E: Está muy bien. ¿Y cómo podés hacer para saber qué dice?

I: Pensar (piensa, mirando a su alrededor). "Mate".

E: ¿Puede ser que diga "mate"?

I: Porque tiene la eme. Me parece que no es porque no está la te. Mirá (pronuncia) mmaaa tteee. Lleva la te. Entonces no sé.

En el ejemplo presentado, Inés recurrió, para la lectura, a una estrategia de análisis fonológico que

Tabla 8

Escritura de palabras por parte de Inés en el mes de junio

\begin{tabular}{lll}
\hline Palabra dictada & Escritura & Pronunciación \\
\hline mesa & MSA & I: me sss aaa \\
tarta & TARA & I: taaaa rrrrr taaaa \\
madera & MDERA & I: ma ma (M) (silencio) Estoy buscando la DE. Cómo es la DE? \\
& E: la de día \\
& I: ¡ah! (Escribe D) qué era? No me acuerdo \\
& E: madera \\
& I: deee (E) rrrr (R) maderr madera maderr ra (A) \\
\hline
\end{tabular}


resulta más apropiada para la escritura. Inés prolongó los sonidos de la palabra mate, identificó en la palabra oral el fonema /t/ y buscó el grafema correspondiente en la forma escrita. Es posible pensar que, en sus intentos por reconocer palabras, Inés recurría a los conocimientos que había adquirido a partir del intenso trabajo de prolongación de sonidos para la escritura de palabras que se realizaba en el centro comunitario. Ahora bien, esta estrategia no es productiva para leer palabras e Inés no lograba transferir a la lectura las habilidades fonológicas que había desarrollado al escribir. La ineficacia del análisis que Inés hacía sobre la forma gráfica que se le presentaba queda también en evidencia en los siguientes ejemplos. Lectura de la palabra nido:

I: Me parece que ahí dice mi nombre. Porque mi nombre lleva esta (N) y la i. Pero esta i tiene que estar primero.

E: ¿Ahi dice tu nombre?

I: No, porque la de "Damián" no lleva mi nombre. Ya sé. Dejame acordarme. “iIndio!”

En el mes de agosto, al presentársele la palabra uno, Inés planteó:

I: Me parece que ahí dice "uva".

E: Ajá.

I: ¿Por qué siempre decís ajá?

E (duda): Para que sepas que te escuché.

I: Pero ahi no dice eso. Es que yo me imagino porque está la U y la N, pero para que diga "uva" tiene que estar la va antes de la $u$.

E: Después de la u.

I: Sí, eso quería decir.
E: Entonces ¿dice "uva” o no?

I: Más o menos.

E: Es una palabra parecida a “uva”. ¿Cómo podés saber qué dice?

\section{I: Mirando.}

E: A ver, mirá.

I: "Lunes".

E: ¿Dice "lunes"?

I: Es que pienso nomás. Pero no está la ele.

La capacidad analítica de Inés contrastaba con sus dificultades para el reconocimiento de palabras. El análisis de las palabras erróneamente pronunciadas revela que no se trataba de respuestas al azar sino que, en todos los casos, sus respuestas presentaban similitudes con el input. Este desempeño sugiere que Inés había realizado un análisis visual detallado de palabras familiares como indio y lunes, análisis que le permitió retener parte de los grafemas que las conforman. Sin embargo, esta estrategia de naturaleza visual no resultó productiva para la lectura de palabras desconocidas. Ante la imposibilidad de reconocer las palabras Inés intentaba "pensar", "acordarse", "imaginar".

La modificación introducida en la propuesta de enseñanza de la lectura en el centro comunitario al que asistía resultó tardía para Inés. Antes de que se comenzara a trabajar sistemáticamente en la enseñanza de la recodificación, Inés empezó a utilizar para la lectura el mecanismo que puede observarse en la Tabla 9.

En las primeras sesiones en las que Inés utilizó este mecanismo, tal como se ve en el caso de las palabras sol y árbol, el procedimiento se superponía con la estrategia de reconocimiento por pista

Tabla 9

Lectura de palabras por parte de Inés en el mes de agosto

\begin{tabular}{ll}
\hline Palabra presentada & Lectura \\
\hline gato & I: la gue con la a, ga. La te con la o, to ga to \\
papá & I: la pe con la a, pa. La pe con la a, pa pa pa \\
árbol & I: la a con la erre, ra. Parece ramo. La a con la erre, rrr la be con la o, \\
& bo...llll (queda en silencio) \\
& E: esta es difícil \\
& I: no es difícil. Árbol \\
& E: ¿Cómo sabías? \\
& I: porque está igualito al cartel de la sala \\
\hline
\end{tabular}


Tabla 10

Lectura de palabras por parte de Inés en el mes de octubre

\begin{tabular}{ll}
\hline Palabra presentada & Lectura \\
\hline balde & I: baaa de \\
cueva & I: cu e la. Abuela. \\
& La entrevistadora señala el final de la primera sílaba \\
& I: cu e va \\
limón & I: li mo na. ¿Limonada? \\
& E: de nuevo \\
& I: li món \\
\hline
\end{tabular}

fonética que usaba anteriormente. Pero tres semanas más tarde Inés lograba recodificar correctamente palabras de dos sílabas con estructura CV. Las dificultades continuaban cuando se complejizaba la estructura silábica, tal como puede apreciarse en la lectura de las siguientes palabras:

Lectura de la palabra tarta

I: La te con la a, ta. La erre con la te.... ¿Cómo suena la erre con la te?

E: Yo te muestro dónde cortar (tapa el final de la palabra, dejando al descubierto la sílaba "tar")

I: la te con la a, ta. ¿Y la erre con quién va?

Lectura de la palabra aro

I: Ahí (señala la A) no puedo leer porque está sola. Tiene que haber otra asi la conjunto.

Si bien los datos del presente trabajo no resultan suficientes para especificar los procesos cognitivos involucrados en el uso de esta estrategia, es posible hipotetizar que al nombrar las letras constituyentes de la sílaba que procuraba reconocer, Inés evocaba las sílabas de un repertorio adquirido previamente.

A fines de octubre Inés leía con precisión, aunque muy lentamente, palabras de hasta cuatro sílabas de estructura CV. Las dificultades persistían al leer palabras con una estructura silábica más compleja (ver Tabla 10).

El ejemplo presentado, correspondiente a finales del mes de octubre, muestra la combinación de estrategias utilizadas ante las palabras que le costaba recodificar. De las 12 palabras presentadas en esa sesión, 2 fueron leídas correctamente. El análisis de las 10 lecturas erróneas reveló que 6 fueron no-palabras (como en el caso de calora), 2 fueron palabras (pateo por pasto) y en otros dos casos (cueva y limón), ante la producción de una no-palabra, Inés evocaba una palabra que compartiera algunos fonemas con la pronunciación que había obtenido.

$\mathrm{Al}$ finalizar el año, pudo reconocer 17 de los 32 ítemes de la prueba de lectura de palabras, siendo la media grupal de 18. El 61\% de las lecturas erróneas dieron lugar a palabras. De las palabras correctamente leídas, el 89\% tenía una estructura CV en todas las sílabas. Inés pudo leer correctamente sólo 2 de 20 pseudopalabras que se le presentaron.

\section{Conclusiones}

El presente trabajo tuvo por objeto estudiar en profundidad el proceso de adopción y modificación de estrategias de lectura y de escritura en niñas de NSE bajo hablantes de español y explorar la relación entre este proceso y las situaciones de enseñanza de las que participaron las niñas.

El patrón de aprendizaje conformado por las estrategias de lectura y de escritura coincidió en buena medida con los resultados obtenidos en trabajos previos realizados en español. Al igual que en otros estudios, se encontró evidencia de la escasa incidencia de una estrategia no analítica en español (Borzone, 1997; Signorini, 1997). Las niñas sólo utilizaron una estrategia logográfica para escribir algunas palabras en los momentos iniciales del aprendizaje, pero rápidamente adoptaron procedimientos analíticos, aunque parciales. También al leer utilizaron tempranamente una estrategia analítica parcial.

Al mismo tiempo, los resultados de este estudio aportan evidencia que complementa la obtenida en estudios previos (Diuk, Signorini \& Borzone, 2003). En dichos trabajos se había observado que los ni- 
ños de NSE bajo hacían uso de una estrategia incompleta de recodificación fonológica y que buena parte de los errores que producían al leer palabras consistía en pronunciar otras palabras que compartían algunas grafías con el estímulo presentado. Este desempeño permitía inferir una tendencia a apoyarse en el conocimiento léxico para completar la recodificación parcial de las palabras.

El recurrir a información fonológica y semántica para identificar palabras puede ser interpretado en el marco del modelo interactivo-compensatorio de Stanovich (2000). Este investigador sostiene que las palabras son sintetizadas en base a información que proporcionan simultáneamente distintas fuentes de conocimiento (semántica, sintáctica, fonológica y ortográfica). Una limitación en cualquiera de las fuentes de conocimiento da lugar a una mayor demanda a otras fuentes de conocimiento, independientemente de su nivel en la jerarquía de procesamiento. En consecuencia, los malos lectores podrían compensar las dificultades que experimentan en el procesamiento fonológico apelando a información léxica.

Ahora bien, en los trabajos previos en los que se identificó una estrategia de recodificación con apoyo léxico se evaluó a los niños al finalizar el ler año escolar. El presente estudio permitió explorar este proceso a lo largo de todo el año y mostró que durante varios meses ambas niñas reconocían palabras mediante el uso de pistas fónicas para seleccionar palabras de su léxico mental, produciendo sistemáticamente respuestas que eran palabras diferentes de las presentadas. En este sentido, el recurso a información léxica no complementó el proceso de recodificación fonológica sino que lo sustituyó. El desempeño observado correspondía al de un proceso detenido en una estrategia analítica parcial.

Ehri (1991) sostiene que la transición entre una estrategia analítica parcial y una estrategia analítica completa es cuantitativa y no cualitativa y que se produce en forma gradual. Los niños pasan de apoyarse solamente en la primera o la última letra de la palabra a atender a un número cada vez mayor de grafemas. En el presente estudio, sin embargo, no se observó en la lectura la transición gradual prevista, aunque sí se la encontró en el aprendizaje de la escritura. En el curso de unos pocos meses las niñas pasaron de utilizar una estrategia semifonética a poder utilizar una estrategia fonética al escribir, produciendo un gradual completamiento de las escrituras. En la lectura, sin embargo, ambas niñas, durante varios meses, no evidenciaron progreso alguno. El desarrollo de las habilidades de conciencia fonológica implícito en el avance de la escritura no se evidenciaba en la lectura.

Este resultado es inesperado dado que existe evidencia en español de un desarrollo rápido de la recodificación fonológica. Así por ejemplo, Cuetos (1989) encontró en niños españoles entre 5 y 6 años un desarrollo de la recodificación fonológica mucho más rápido que el que experimentan niños que aprenden a leer en lenguas de ortografía opaca tales como el inglés.

Resulta sumamente relevante, en consecuencia, intentar identificar las causas de esta ausencia de progreso en las niñas estudiadas. Por un lado, se podría pensar que la asincronía en el desarrollo de la lectura y la escritura se relaciona con las demandas que cada una de estas habilidades plantea al niño. Para leer una palabra mediante una estrategia analítica el niño debe identificar cada grafema, establecer la asociación con el fonema correspondiente, ensamblar la sílaba y retenerla mientras recodifica la sílaba siguiente. Debe luego ensamblar las sílabas entre sí para reconocer la palabra. Este proceso es más demandante que el que tiene lugar al escribir, cuando el niño retiene en la memoria una palabra conocida mientras la analiza, identifica los fonemas que la componen y establece las correspondencias.

Ahora bien, la dificultad relativa de los procesos de lectura y escritura no parece poder explicar el desempeño de las niñas participantes en este estudio. No se trataba de que las niñas no fueran eficaces en el proceso de recodificación sino que desconocían el mecanismo. Por otra parte, el nivel de conciencia fonológica y la capacidad de memoria puestos de manifiesto por Natalia al obtener la palabra leona adicionando el fonema /a/ a la palabra león, sugieren que la dificultad de la niña no estaba localizada en el desarrollo de las habilidades básicas requeridas para el uso de una estrategia analítica en la lectura.

Cabe entonces considerar la posibilidad de que el patrón de desempeño de las niñas estuvo relacionado con la intervención pedagógica de la que participaron. En esta intervención, se ejercitaba sistemáticamente la segmentación fonológica y la escritura, pero no se realizaba un trabajo analítico similar para la lectura. Cardoso-Martins (2001) ha mostrado cómo las estrategias utilizadas por los niños están relacionadas con el método de enseñanza. En un estudio realizado con niños hablantes de portugués, esta investigadora encontró que, a diferencia de los niños cuyos docentes utilizaban un método fónico, los niños a los que se enseñaba mediante un método global no mostraron evidencia de hacer uso de una 
estrategia de recodificación fonológica hasta tanto se comenzó a introducirlos en las relaciones grafemafonema.

En el mismo sentido, el desempeño de Inés y Natalia sugiere que cuando la enseñanza no proporciona a los niños los conocimientos y habilidades necesarios para avanzar hacia la recodificación fonológica, este aprendizaje no siempre tiene lugar. Más específicamente, el desempeño de Natalia muestra que no es suficiente con el conocimiento de las correspondencias sino que en ciertos casos, se requiere, además, de la enseñanza explícita del mecanismo de recodificación mediante el modelado del ensamblaje de la pronunciación.

Estos resultados sugieren que la tendencia a la recodificación fonológica lexicalizada identificada en estudios previos es, probablemente, el resultado de la implementación de prácticas inadecuadas de enseñanza de la lectura.

Las implicancias pedagógicas de estos resultados están en línea con los planteos que diversos investigadores vienen realizando en los últimos años, señalando que los bajos niveles de aprendizaje de los niños que crecen en contextos de pobreza son consecuencia de haberlos privado de instrucciones metafonológicas y fónicas explícitas y sistemáticas (Capovilla \& Capovilla, 2004).

\section{Referencias}

Adams, M. J. (1990). Beginning to read. Thinking and learning about print. Cambridge, Massachusets: MIT Press.

Borzone, A. M. (1997). El proceso de alfabetización en niños pequeños: Diferencias socioculturales. Tesis doctoral no publicada, Universidad de Buenos Aires, Argentina.

Borzone, A. M. \& Diuk, B. (2001). El aprendizaje de la escritura en español: Estudio comparativo entre niños de distinta procedencia social. Interdisciplinaria, 18, 1-33.

Borzone, A. M. \& Signorini, A. (1994). Phonological awareness, spelling and reading abilities in Spanish-speaking children. British Journal of Educational Psychology, 64, 429-439.

Borzone, A. M. \& Signorini, A. (1998). Emergent writing forms in Spanish. Reading and Writing: An Interdisciplinary Journal, 10, 499-517.

Bravo, L., Villalón, M. \& Orellana, E. (2002). La conciencia fonológica y la lectura inicial en niños que ingresan a primer año básico. Psykhe, 11, 175-182.

Capovilla, A. G. \& Capovilla, F. C. (2004). Problemas de lectura e escrita. Sao Paulo, SP: Memnon Edicoes Científicas.

Cardoso-Martins, C. (1995). Sensitivity to rhymes, syllables and phonemes in literacy acquisition in Portuguese. Reading Research Quarterly, 30, 808-828.

Cardoso-Martins, C. (2001). The reading abilities of beginning readers of Brazilian Portuguese: Implications for a theory of reading acquisition. Scientific Studies of
Reading, 5, 289-317.

Cuetos, F. (1989). Lectura y escritura a través de la ruta fonológica. Infancia y Aprendizaje, 45, 71-84.

Diuk, B., Borzone, A. M. \& Rosemberg, C. (2000). El fracaso escolar entre los niños de sectores pobres: Una alternativa pedagógica intercultural. Cultura y Educación, 19, 23-33.

Diuk, B., Signorini, A. \& Borzone, A. M. (2003). Las estrategias tempranas de lectura de palabras en niños de 1 er ciclo de E.G.B.: Un estudio comparativo entre niños procedentes de distintos sectores sociales. Psykhe, 12(2), 5162.

Ehri, L. C. (1991). Development of the ability to read words. En R. Barr, P. Mosenthal \& P. D. Pearson (Eds.), Handbook of reading research (Vol. II, pp. 383-417). New York: Longman.

Ehri, L. C. (1999). Phases of development in learning to read words. En J. Oakhill \& R. Beard (Eds.), Reading development and the teaching of reading. A psychological perspective (pp. 79-108). Oxford, England: Blackwell.

Ellis, N. C. (1994). Longitudinal studies of spelling development. En G. D. A. Brown \& N. C. Ellis (Eds.), Handbook of spelling (pp. 155-187). Chichester: John Wiley \& Sons.

Fletcher-Flinn, C. M. \& Thompson, G. B. (2000). Learning to read with underdeveloped phonemic awareness but lexicalized phonological recoding: A case study of a 3year-old. Cognition, 74, 177-208.

Frith, U. (1985). Beneath the surface of developmental dyslexia. En K. E. Patterson, J. C. Marshall \& M. Coltheart (Eds.), Surface dyslexia: Neuropsychological and cognitive studies of phonological reading (pp. 67-84). London: Erlbaum.

Frith, U., Wimmer, H. \& Landerl, K. (1998). Differences in phonological recoding in German- and English-speaking children. Scientific Studies of Reading, 2, 31-54.

Goswami, U., Gombert, J. E. \& Fraca da Barrera, L. (1998). Children's orthographic representations and linguistic transparency: Nonsense word reading in English, French and Spanish. Applied Psycholinguistics, 19, 19-52.

Perfetti, C. A. (1992). The representation problem in reading acquisition. En P. B. Gough, L. C. Ehri \& R. Treiman (Eds.), Reading acquisition (pp. 145-174). Hillsdale, NJ: Erlbaum.

Siegler, R. S. (1991). Children's thinking. Englewood Cliffs, NJ: Prentice Hall.

Signorini, A. (1997). Word reading in Spanish: A comparison between skilled and less skilled beginning readers. Applied Psycholinguistics, 18, 319-344.

Signorini, A. (1999). Del habla a la escritura: El procesamiento fonológico en la lectura inicial. Tesis de Doctorado no publicada, Universidad Nacional de La Plata, Argentina.

Signorini, A. \& Borzone, A. M. (1996). Las habilidades metafonológicas, la lectura y la escritura en niños de cinco años. Lenguas Modernas, 23, 71-95.

Signorini, A. \& Piacente, T. (2001). Adquisición de la lectura en español: Las habilidades de procesamiento de palabras en lectores iniciales. Revista Irice, 15, 5-29.

Stanovich, K. E. (2000). Progress in understanding reading reading: Scientific foundations and new frontiers. Nueva York-Londres: The Guilford Press.

Steffler, D. J., Varhagen, C. K., Friesen, C. K. \& Treiman, R. (1998). There's more to children's spellings than the errors they make: Strategic and automatic processes for one- 
syllable words. Journal of Educational Psychology, 90, 492-505.

Wimmer, H. (1993). Characteristics of developmental dyslexia in a regular writing system. Applied Psycholinguistics, 14, 1-33.

Fecha de recepción: Noviembre de 2005.

Fecha de aceptación: Diciembre de 2006.
Wimmer, H. \& Goswami, U. (1994). The influence of orthographic consistency on reading development: Word recognition in English and German children. Cognition, 51, 91-103. 